\title{
The Ontological Form of Tropes
}

\section{Refuting Douglas Ehring's Main Argument against Standard Trope}

\author{
Nominalism
}

Jani Hakkarainen and Markku Keinänen, University of Tampere

\begin{abstract}
According to standard trope nominalism, there are simple tropes that do not have parts or multiply distinct aspects. Douglas Ehring's reductio ad absurdum against this standard view concludes that there are no simple tropes. In this paper, we provide a response to Ehring defending the standard view. Ehring's argument may be refuted by (1) distinguishing the ontological form of tropes from their contribution to the ontological content of the world, and (2) construing tropes as having primitive identity. At the same time, standard trope nominalism is elaborated on by distinguishing between ontological form and content, for which there are also independent reasons.
\end{abstract}




\section{Introduction}

In this paper, we respond to Douglas Ehring's most convincing argument against standard trope nominalism in his recent book (2011). Such a response was missing in the literature. ${ }^{1}$ Using a reductio ad absurdum of standard trope nominalism, Ehring concludes that there are no simple tropes - that is, tropes that lack parts or multiple, non-identical intrinsic "aspects". This clearly contradicts the standard view, according to which there are simple tropes.

Our claim is that Ehring's argument against the simplicity of standard tropes is not sound; it is premised on an incorrect principle. Therefore, Ehring's reductio ad absurdum against the standard view fails. The incorrect principle in the argument is that if a and $b$ are related by arbitrarily different internal relations, then $a$ and $b$ are not simple. This involves a hidden premise: entities in distinct arbitrarily different internal relations have parts or multiple, non-identical aspects. Arbitrarily different internal relations are internal relations that vary independently from each other. In his argument, Ehring shows that exact resemblance and numerical distinctness among tropes are arbitrarily different internal relations. On this basis, he concludes by the incorrect principle that exactly resembling and numerically distinct tropes are not simple.

We shall show that the principle is incorrect for two main reasons. First, the ontological content of the world in trope nominalism should be distinguished from the ontological form of tropes. The holding of the exact resemblance of tropes, which describes the ontological content, is independent of the holding of the numerical distinctness of tropes, which spells out their ontological form, and vice versa. So, our response to Ehring draws on the distinction between ontological form and ontological content, employed, for instance, by E.J. Lowe, Kevin Mulligan, and Barry Smith. Actually, our response to Ehring not only defends standard trope nominalism against criticism; we elaborate on standard trope nominalism using the ontological content/form distinction. Our second

\footnotetext{
${ }^{1}$ For responses to a similar but distinct criticism of the standard view, see Maurin (2005).
} 
main reason for denying Ehring's principle is that the identity of tropes is primitive: tropes do not have informative and non-circular identity conditions provided by distinct entities.

Ehring's motivation to argue against standard trope nominalism is due to his own non-standard, natural-class view of tropes (2011, ch. 6). He explains the resemblance of objects by the primitive relation of being a member of a natural class holding of tropes - in contrast to the standard explanation in virtue of the existence of resembling particular thin natures that are borne by objects. ${ }^{2}$ So, Ehring does not identify tropes with thin natures, which is essential to the standard view held by D.C. Williams (1953), Keith Campbell (1990), Peter Simons (1994), Anna-Sofia Maurin (2002), and us (2010, 2014, Keinänen 2011), among others. According to the standard view, tropes bestow only a single property on the objects that bear them, for example a $-e$ charge on each electron and an $e$ charge on positron. The standard view also involves the tenet that there are mereologically simple tropes.

The paper has four parts. In the first section, we discuss Ehring's reductio and judge his argument to the result that exact resemblance and numerical distinctness among tropes are arbitrarily different internal relations. The second section is devoted to the characterization of the ontological content/form distinction, which we argue for in the fourth section. We respond to Ehring's reductio in the fourth section, which also concludes the paper.

\section{Ehring's Argument}

Ehring's argument targets the statement in the standard view that tropes do not "have distinct particularity-giving and nature-giving components" $(2011,176)$. If they had these components, the

\footnotetext{
${ }^{2}$ Standard trope nominalism provides a neat and parsimonious solution to the problem of universals - the metaphysical explanation of the resemblance of objects. Trope-bundle theory, in which objects are composed of nothing but particular thin natures, is also able to account for the bearing of properties by objects well (e.g. Campbell 1990, ch.1; Keinänen 2011, Keinänen \& Hakkarainen 2010 and 2014). For a review of the other advantages of trope nominalism, see Ehring 2011, ch. 2.
} 
components would render tropes complexes consisting of a particularity-giving component and a particular exemplification of a property universal (ibid.). For example, $e$ charge tropes would have non-identical particularity-giving and nature-giving components, the latter of which would be the exemplifications or instantiations of the $e$ charge property universal. By contrast, according to the standard view, each $e$ charge trope is simple: it "lacks parts and lacks multiple, non-identical intrinsic 'aspects"” (ibid.). ${ }^{3}$ The standard view identifies each simple standard trope with a thin nature (e.g. $e$ charge), which is a particular, individual entity.

Ehring offers three versions of his argument. The last version, which he considers the most convincing, is as follows:

If $a$ and $b$ are related by arbitrarily different internal relations then $a$ and $b$ are not simple. Trope $t_{1}$ is numerically different from trope $t_{2}$ and trope $t_{1}$ resembles trope $t_{2}$ exactly. Resemblance among Campbellian tropes [identified with thin natures] and the difference relation are internal relations that are arbitrarily different than each other. Hence, $t_{1}$ and $t_{2}$ are not simple. (Ehring 2011, 179-80; cf. Hochberg 2004, 23-4; Moreland 2001, 70-1; Maurin 2005, 138)

In order to see that Ehring's argument has the form of a reductio ad absurdum, it is useful to formulate it as a series of steps:

1) Suppose that trope $t_{1}$ and trope $t_{2}$ are simple standard tropes: ${ }^{4}$ they lack parts and multiple, distinct $^{5}$ intrinsic aspects, are identified with thin natures, and are particulars.

\footnotetext{
${ }^{3}$ For present purposes, it is sufficient that intrinsicness means that intrinsic $x$ is possessed by an object independently of the existence of other objects that are not its proper parts. About "multiple, non-identical intrinsic 'aspects", Ehring says that it "is unnecessary if it is assumed that a property of something is a part of that thing. However, since some will find this assumption to be questionable and since it is at least an open question whether something that lacked parts might have more than one intrinsic property, I have included the second conjunct" (Ehring 2011, 176-7). We shall follow Ehring here and talk about aspects in addition to parts in order to cover those possible views in which non-identical intrinsic aspects are not parts (they could be modes, which are not parts of objects, for instance).

${ }^{4}$ We talk about "standard tropes" instead of "Campbellian tropes" because the standard view is not unique to Campbell.

${ }^{5}$ Henceforth, we shall not use Ehring's terms "numerical difference" or "non-identity" but "distinctness", which is standard terminology meaning the negation of numerical identity.
} 
2) Suppose: "Trope $t_{1}$ is numerically different from trope $t_{2}$ and trope $t_{1}$ resembles trope $t_{2}$ exactly."

3) "Resemblance among Standard tropes and the difference relation are internal relations that are arbitrarily different than each other."

4) Hidden premise: entities in distinct arbitrarily different internal relations are not simple: they have parts or multiple, distinct intrinsic aspects.

5) "If $a$ and $b$ are related by arbitrarily different internal relations then $a$ and $b$ are not simple."

6) "Hence, $t_{1}$ and $t_{2}$ are not simple": they have parts or multiple, distinct intrinsic aspects.

7) Hence, there is a contradiction [between steps 1 and 6].

8) Ergo, standard trope nominalism is false. (Ehring 2011, 179-80)

In our reformulation of Ehring's argument, we have inserted the missing premise between steps 3 and 5. We take it to be obvious that this hidden premise is needed for the argument to be sound. Otherwise, it would not follow that the relata of arbitrarily different internal relations are not simple.

Initially, our response is that the consequence of Ehring's argument does not follow. Obviously, the key step in the argument is step 3: distinctness and exact resemblance among simple standard tropes are arbitrarily different internal relations. What does this mean? Let us begin with Ehring's characterization of arbitrarily different relations (internal or not). Distinctness and exact resemblance among all simple standard tropes are arbitrarily different relations if the following two conditions are satisfied:

1. Realization of distinctness/exact resemblance among any simple standard tropes does not metaphysically necessitate the realization of exact resemblance/distinctness. ${ }^{6}$

\footnotetext{
${ }^{6}$ Henceforth, we shall drop "metaphysical" qualifying modal terms. We shall mean metaphysical modalities, unless otherwise noted.
} 
2. Not every variation with respect to distinctness/exact resemblance necessitates a variation with respect to exact resemblance/distinctness. ${ }^{7}$ (Ehring 2011, 177-8)

Ehring is correct in stating that distinctness and exact similarity among simple standard tropes differ arbitrarily $(2011,178)$. The second condition about variation is satisfied almost trivially in the case of simple tropes in the standard view. First, since simple standard tropes are identified with thin natures, their exact resemblances cannot vary. Secondly, if distinct and exactly resembling tropes were identical, they would remain exactly resembling (due to being identical). Therefore, not every variation with respect to one of these relations (distinctness or exact resemblance) necessitates a variation with respect to another (exact resemblance or distinctness).

The first condition is also satisfied by simple standard tropes: the realization of distinctness/exact resemblance among any of these tropes does not necessitate the realization of the other. We can see this by modifying Ehring's presentation slightly and considering three simple standard tropes, $t_{1}, t_{2}$, and $t_{3}$. Suppose first that they are distinct from each other. Clearly, this does not necessitate exact resemblances among $t_{1}, t_{2}$, and $t_{3}$. It is possible that $t_{1}$ and $t_{2}$, for instance, exactly resemble each other but $t_{1}$ and $t_{3}$ do not ( $t_{1}$ and $t_{2}$ are, for example, $e$ charge tropes, whereas the third is $t_{3}$ is an $e / 3$ trope). On the other hand, we may begin with the assumption that simple standard tropes $t_{1}$ and $t_{2}$ exactly resemble each other. This does not necessitate distinctness or identity between $t_{1}$ and $t_{2}$. It is possible, given their exact resemblance, that these entities are distinct or that they are identical. In other words, the identity of indiscernibles does not hold true of standard tropes. According to Ehring's definition of being particular, particulars do not conform to the identity of indiscernibles $(2011,35)$. Hence, tropes are particulars in Ehring's sense.

We can therefore conclude that distinctness and exact resemblance among simple standard tropes are, indeed, arbitrarily different relations. But are they arbitrarily different internal relations? In

\footnotetext{
${ }^{7}$ We adjust Ehring's formulation slightly to allow the necessitation of distinctness from the absence of exact resemblance.
} 
characterizing internal relations, Ehring follows Armstrong: “A relation is internal [...] when given certain terms with certain natures, the relation must hold between the terms. It holds in every possible world that contains these terms and where these terms have these natures." (Armstrong 1989, 43; quoted by Ehring 2011, 179)

In this paper, we employ Ehring's Armstrongian terminology in this respect for the sake of the argument. According to Ehring, distinctness and exact resemblance among all simple standard tropes are internal relations. In standard trope nominalism, exact resemblances of simple tropes obtain in every possible world where the related tropes exist. Their holding is necessary, given the existence of their relata as certain natures, because simple tropes are identified with certain thin natures. Distinctness among simple standard tropes is also an internal relation because, arguably, distinctness is necessary to distinct entities. Distinctness holds in every possible world where its relata exist; we do not actually need the additional condition about natures in the case of distinctness. ${ }^{8}$

\section{Distinction between Ontological Content and Ontological Form}

Ehring is correct in stating that exact resemblance and distinctness among any simple standard tropes are arbitrarily different internal relations. Nevertheless, step 5 is not correct about these relations. It does not follow from the fact that simple standard tropes $a$ and $b$ are related by distinctness and exact resemblance that $a$ and $b$ are not simple. Recall that there is a hidden premise at this point in Ehring's argument: entities in distinct arbitrarily different internal relations are not simple. So, they have parts or multiple, distinct intrinsic aspects. Step 5 is incorrect because this hidden premise is false.

\footnotetext{
${ }^{8}$ According to Moore (1960 [1922]), internal relations are essential to the identity of their relata and hold necessarily if their relata exist, cf. Campbell (1990,112). Due to holding necessarily, given the existence of the relata, exact similarity and distinctness among tropes are internal relations in this strong sense. They are also internal relations in Kevin Mulligan's characterization: "a relation is internal with respect to objects, a, b, c etc., just if, given a, b, c etc., the relation must hold among and of these objects" $(1998,344)$.
} 
It is false because distinct internal relations may differ arbitrarily without rendering their relata non-simple, due to the difference between what E.J. Lowe's calls "ontological content" and “ontological form" $(2006,48) .{ }^{9}$ Elaborating on Lowe's distinction, we shall argue in Section 4 that this is precisely the case with exact resemblance and distinctness among simple standard tropes. First, however, we will begin by explicating the distinction in this section.

Smith (1981), together with Kevin Mulligan (Smith and Mulligan 1983), brought the distinction between ontological content and ontological form into the contemporary discussion from Edmund Husserl (1859-1938). This distinction is analogous to the logical contrast between the non-logical content of a proposition and its logical form (Smith 1981; Smith and Mulligan 1983; Lowe 2006, 48). ${ }^{10}$ It is to be insisted, however, that ontological and logical form should not be conflated, because the former is the form of being in general, whereas the latter is much more restricted: it is the form of truth-bearers, assuming that truth-bearers are entities (Smith and Mulligan 1983, 73; Smith 2005, ch. 5; Lowe 2006, 48).

It is not necessary for our present purposes to give a comprehensive account of the distinction between ontological content and ontological form, which is no easy task. As a response to Ehring, it suffices to characterize this distinction. This can be achieved by employing three primitive concepts: existence, nature, and manner. By combining existence and manner, we get a fourth concept, which is crucial for characterizing ontological form: manner of existence. The concepts of existence, nature, and manner of existence may be characterized and introduced by way of examples, but the first two and manner cannot be given an eliminative non-circular definition because they are primitive concepts.

One may consider anything one believes to exist, a human body for instance. In one's view, every such entity adds to the ontological content of the world - what beings there are. This is "ontology" in

\footnotetext{
${ }^{9}$ Cf. Smith and Grenon 2004.

${ }^{10}$ Cf. Mulligan 1998 (343) for criticism of drawing a clear distinction between the logical and non-logical.
} 
Quinean terms; one makes an "ontological commitment". Most likely, the belief in this entity also involves a belief about its nature: a belief whose content is a description of the entity (e.g. the body weighs $100 \mathrm{~kg}$ ). ${ }^{11}$ Properties such as quantities (e.g. rest masses and electric charges) and perhaps qualities (e.g. the shape of a cube) provide paradigmatic examples of natures, which Campbell (1990, 6) characterizes as the intrinsic (i.e. non-relational) characters of entities. For instance, in the classic substance-property ontology, the nature of a substance consists of its properties (ibid.). Presumably, natures divide entities into kinds (e.g. masses, cubes, etc.). ${ }^{12}$

By contrast, if one also believes that an entity (e.g. the human body) exists as being numerically identical (it is the same with itself), one holds a belief about the manner of the existence of the entity, a belief whose content is a description of its existence. ${ }^{13}$ The concept of identity does not describe the non-relational character of the identical entity, which determines its kind, but its manner of existence. ${ }^{14}$ The crucial point is that one does not believe in any entity in addition to the entity being identical (e.g. the human body); rather, this is a belief about the manner in which the entity exists as a constituent of the world (e.g. that the human body is the same with itself). The belief about the identity of the entity therefore concerns the ontological form of the world.

Manners of existence may be said to be formal features of entities. ${ }^{15}$ Formal features are not entities, for instance additional tropes. ${ }^{16}$ So, formal features cannot be said to be identical to anything, pace Ehring, because the relata of the relation of identity must be entities (Ehring 2011, 183). Thus, formal

\footnotetext{
${ }^{11}$ The sentence that there is something does not entail that this entity has some nature. It is at least logically possible that there are entities without any essential nature. The advocates of bare particulars believe in such entities.

12 The sense of "nature" employed here should not be conflated with "essence", since the nature of an entity is not necessarily essential to it. "Nature" has a larger extension than "essence". Moreover, we are not making any assumption about the ontological priority of existence and nature (cf. the age old-debate about the ontological priority of existence and essence). They might be ontologically on a par.

${ }^{13}$ In principle, it seems unnecessary for believing in the existence of an entity to hold that it has identity, since it appears to be possible to believe in the existence of something as a mere unity. So, it does not seem to be the case that every entity must have determinate identity-conditions (cf. Lowe 2006, 75).

${ }^{14}$ It is to be insisted that the concept of manner of existence does not entail that "existence" has more than one meaning. We take it to be univocal. However, entities may exist in various different manners (cf. below).

${ }^{15}$ We use "feature" in an ontologically non-committal way. Therefore, we use it instead of "property" for manners of existence.

${ }^{16}$ Plausible examples of tropes, from the point of view of our a posteriori scientific knowledge, are basic physical quantities (e.g. determinate charges, rest masses, and spin quantum numbers).
} 
features are not identical to or distinct from the entity having that feature, an entity of some category. Rather, formal features are manners of existence of entities.

Arguably, other examples of formal features than identity are being one (unity), being an individual (being identical and being one), being a particular (being a universal for the realist), being a part, being a whole, being dependent, and being a dependee. Their concepts contribute to the description of the manner of the existence of entities as one, identical and one, as particular, as a part, a whole, dependent, and a dependee.

The distinction between ontological content and ontological form, put explicitly in terms of the representation of the world (a metaphysical view or theory), may be explicated as follows: the ontological content side of the representation consists of its ontological commitments and possibly descriptions of the natures of the commitments that correspond to entities. Part of this side may be, for instance, that there is an $e$ charge. By contrast, the ontological form side of the representation describes the manner of existence of the entities. The $e$ charge, for example, is believed to exist as identical. If this belief represents the world correctly, identity is a formal feature of the $e$ charge entity in contrast with its nature, that is, $e$ charge. This formal feature is described by the predicate term "is identical" applying to the entity. In Lowe's terms, it is a "formal ontological predicate" (Lowe 2006, 193). ${ }^{17}$ The nature is described by the ontological predicate "is $e$ charge". Formal ontological predicates describe formal features of entities, whereas ontological predicates describe their nature.

Accordingly, ontological relational predicates contribute to the description of the nature of entities and formal ontological relational predicates contribute to the description of the formal features of entities. Ontological relations and formal ontological relations, respectively, are relations holding

\footnotetext{
${ }^{17}$ While the set of ontological commitments matches "ontology" in Quinean terms, ontological form does not equate with Quine's "ideology". According to Quine, ideology of a theory is the "stock" of its predicates and occasion sentences (Quine 1983, 501). Not all predicates or concepts of a metaphysical theory need to be formal ontological.
} 
among entities. ${ }^{18}$ Exact resemblance is an ontological relation, whereas identity is a paradigmatic example of a formal ontological relation. ${ }^{19}$ Their corresponding predicate terms contribute to the description of the nature of entities and their formal features.

\section{Argument for the Ontological Content/Form Distinction}

There are independent reasons for the distinction between ontological content and ontological form. The main independent reason is that this distinction satisfies the theoretical virtue of modesty (Kriegel 2013, 20, cf. Quine and Ullian 1978, 68ff.). When the distinction is embraced, there is no need to make ontological commitments in the case of formal features. So, one's ontological commitments are modest; one makes fewer claims when one embraces the concept of ontological form. At the same time, there is no decrease in the explanatory power of the theory involving the distinction; being identical, being one, being particular, etc., are accounted for by construing them as formal features of entities. One arrives at the same result with weaker assumptions.

Furthermore, the theory involving the ontological content/form distinction does not suffer from a serious problem of its main contender. This contender is the nominalist reification view that formal features are particular entities. In the context of trope nominalism, the following infinite regress about the formal feature of particularity ensues for the alternative view:

1) Suppose the truth of nominalism and the existence of tropes: there are only particulars and there are tropes.

2) Suppose that there is standard trope $x$ that is identified with $e$ charge.

\footnotetext{
${ }^{18}$ In addition to identity, plausible examples of formal ontological relations include distinctness, mereological relations, and relations of existential dependence (such as rigid and generic dependence). They determine how an entity exists as a constituent of the world (e.g. as a part of other entities) or how it can exist as a constituent of the world (relations of existential dependence), cf. Smith and Mulligan (1983).

${ }^{19}$ Consequently, we do not endorse the tenet that tropes resemble one another in respect of their formal features. This suspension undermines Ehring's second argument against standard trope nominalism. The second argument is premised on the assumption that tropes resemble one another in respect to their particularity (Ehring 2011, 182).
} 
3) Thus, trope $x$ is particular [from steps 1 and 2; "the is of predication", in contrast to step 2].

4) Suppose that the feature of $x$ being particular is reified.

5) Thus, there is entity $y$ that is identified with the particularity of $x$ [from steps 3 and 4].

6) Thus, entity $y$ is distinct from $x$ [from steps 2 and 5; because $x=e$ charge].

7) Thus, distinct entity $y$ is particular [from steps 1,5 , and 6].

8) Suppose that the feature of $y$ being particular is reified.

9) Thus, there is entity $z$ that is identified with the particularity of $y$ [from steps 7 and 8].

10) Thus, entity $z$ is distinct from $y$ and $x$ [from steps 2, 5, and 9; because $y=$ the particularity of $x$ and $x=e$ charge].

11) Thus, distinct entity $z$ is particular [from steps 1, 9, and 10], and so on, ad infinitum.

Two remarks about this argument are in order. First, step 6 follows from a highly plausible principle in addition to steps 2 and 5 (alike in the case of step 10, and so on and so forth). This principle is the non-identity of distinguishables (if something true of $x$ is false of $y, x$ is not identical with $y$ ), which is the contrapositive of Leibniz's Law (the indiscernibility of identicals). Trope $x$ and entity $y$ are not identical because something is true about $x$ that is not true about $y-$ that $x=e$ charge.

Secondly, the argument does not presuppose that $y$, $z$, etc., are tropes. In principle, they may be particulars of some other category (e.g. a bare particular or a set). Still, the particularity of each of these entities is reified. Assuming that one of these entities is a set does not halt the regress because sets are not their own members. For instance, if $y$ is taken as a set, that is, as the set of particulars, it does not stop the regress since this set itself is particular, which cannot be coherently accounted for by the set itself.

This infinite regress is vicious for the reason that it represents an explanatory failure about a general metaphysical problem (Hakkarainen \& Keinänen 2016, 250; cf. Bliss 2013). Step 3 may be understood as a metaphysical explanandum. So, the initial task of explanation here is to account for the particularity of $x$. The explanans is to postulate entity $y$, which is identified with the particularity 
of $x$. The next explanandum is the particularity of $y$, which is accounted for by the postulation of entity $z$, identified with the particularity of $y$. A pattern emerges. The two explananda are exactly of the same type: the particularity of an entity. This type of explanandum is repeated an infinite number of times. The same type of explanans is equally repeated an infinite number of times: entity ${ }_{n+1}$ is identified with the particularity of entity ${ }_{n}$. So in each step, the explanans involves the explanandum. Hence, nothing at all is explained about the general metaphysical problem - the particularity of an entity (the type of explanandum). No answer is given to the problem at hand, and no step forward is taken.

When particularity is construed as a formal feature, the regress is halted at step 3; the particularity of $x$ is not postulated as an entity. So, construing particularity as a formal feature does not suffer from the same metaphysical explanation failure as postulating particularity as an entity. Moreover, it can tell us in which particularity consists: particularity is existing in a certain manner. This manner of existence can be given a theoretical description. Assuming nominalism, the strongest candidate for the theoretical description of particularity seems to be that particular entities can be exactly similar and distinct, which is also Ehring's characterization of particularity. All in all, embracing the ontological content/form distinction is clearly superior to its main contender, the reification of formal features. For this reason, and to be theoretically modest, holding this distinction is justified.

\section{4. $\quad$ Response to Ehring}

In Section 1, we agreed with Ehring that the relations of exact resemblance and distinctness among simple standard tropes differ arbitrarily. The realization of distinctness/exact resemblance among any simple standard tropes does not necessitate the realization of exact resemblance/distinctness among them, nor does every variation with respect to distinctness/exact resemblance necessitate a variation with respect to exact resemblance/distinctness. 
Next, with the help of the above argument, we shall argue that this does not jeopardize the simplicity of standard tropes. Ehring's hidden premise that entities in distinct arbitrarily different internal relations have parts or multiple, non-identical intrinsic aspects is false. Therefore, his step 5 (If $a$ and $b$ are related by arbitrarily different internal relations, then $a$ and $b$ are not simple) is not correct about simple standard tropes. Rather, simple standard tropes can both be in arbitrarily different internal relations and be simple. Accordingly, it is our task to account for the fact that arbitrarily different internal relations among standard tropes can have truly simple relata. Here the ontological content/form distinction proves useful. First, we will show how it is possible that the internal relations of exact resemblance and distinctness among simple standard tropes differ arbitrarily.

Let us first discuss how the holding of distinctness and identity of thin natures - which simple standard tropes are - is independent from the holding of their exact resemblance. Distinctness and identity describe the ontological form of thin natures, and exact resemblance spells out their contribution to the ontological content of the world. In other words, distinctness and identity are formal ontological relations, whereas exact resemblance is an ontological relation. In our schematic example above, we made an assumption about the ontological content of the world: there are thin natures, that is, the $e$ charges and the $e / 3$ charge. The ontological relation of exact resemblance holds between the $e$ charges but not between an $e$ charge and the $e / 3$ charge. Necessarily, given only that there are the $e$ charges and the $e / 3$ charge, one $e$ charge exactly resembles the other $e$ charge but the $e / 3$ charge does not exactly resemble the $e$ charges (and vice versa).

However, given that the above-mentioned assumption about the holding and non-holding of exact resemblance is correct, it is equally possible that all of these entities are distinct from each other, that " $e$ charges" are actually identical or that the $e$ charges are exactly resembling instances of one and the same $e$ charge property universal. There is nothing in the existence and nature of the $e$ charges and the $e / 3$ charge as abstracted from their formal features that would necessitate any of these specific possibilities. Still the $e$ charges and the $e / 3$ charge must arguably have some formal features in order 
to exist. The holding of exact resemblance among thin natures does not necessitate the holding of distinctness and identity among them. In this sense, the formal ontological relations of distinctness and identity, on the one hand, and the ontological relation of exact resemblance, on the other, are independent of each other. It is a further fact about the actual world that only the following relations of identity hold in the scheme: $t_{1}=t_{1}=e$ charge, $t_{2}=t_{2}=e$ charge, $t_{3}=t_{3}=e / 3$ charge.

In the other direction, the holding of distinctness of simple standard tropes does not necessitate the holding of their exact resemblance. If simple standard tropes are distinct, they may or may not exactly resemble each other. The ontological relation of exact resemblance among simple standard tropes, which describes the ontological content of the world, holds independently from the formal ontological relation of distinctness, which describes the ontological form.

Hence, the holding of exact resemblance of simple standard tropes and the holding of their distinctness are mutually independent. Therefore, these ontological and formal ontological relations can be arbitrarily different, which is the first part of our task of explanation. For the second task, which is to show that the simple standard tropes in these mutually independent relations can indeed be simple, another elaboration on the ontological content/form distinction is required.

In our example, we assumed the further fact that only $t_{1}=t_{1}=e$ charge, $t_{2}=t_{2}=e$ charge, and $t_{3}=t_{3}=e / 3$ charge hold in the scheme. This is in line with the view we have argued for elsewhere that the identity of simple standard tropes is primitive: they do not have informative or non-circular identity-conditions that are given by entities distinct from the tropes (Keinänen \& Hakkarainen 2014). In other words, at least simple standard tropes are identity-independent entities. On this issue, we agree with Campbell (1990, 55ff.). Our reason to hold this view is, in a nutshell, that it is the only coherent position about the identity of simple standard tropes that does not rule out, as it ought not to, the possibility of co-located exactly similar simple standard tropes (pace Maurin 2014, Ch. 2.3). Moreover, if the identity-conditions of simple standard tropes were given by objects, a vicious circularity in identity-conditions would threaten trope-bundle theory, as Lowe (1998, 205-9) argues. 
In the trope-bundle theory, objects are ultimately composed of simple standard tropes, which give the identity-conditions for objects. So, it would be circular to hold that objects, in turn, give identityconditions for simple standard tropes. Arguably, this circularity in identity-conditions would be vicious.

Therefore, it is a primitive fact in our and Campbell's trope theory that only $t_{1}=t_{1}=e$ charge, $t_{2}=t_{2}=e$ charge, and $t_{3}=t_{3}=e / 3$ charge hold in our example; the holding of these identity relations is not determined by any entity distinct from $t_{1}, t_{2}$, and $t_{3}$. However, the fact that these and only these identity relations hold in the scheme necessitates the holding of another formal ontological relation, distinctness: $t_{1} \neq t_{2} \neq t_{3}$. Thus, the holding of $t_{1} \neq t_{2} \neq t_{3}$ is not determined by any entity distinct from these simple standard tropes either. The first two of these entities are also the sole relata of their exact resemblance. Ex hypothesi, $t_{1}$ and $t_{2}$ are thin natures and thin natures may resemble each other exactly without being complex. Consequently, it is possible that no entity distinct from $t_{1}, t_{2}$, and $t_{3}$ determines the mutually independent holding of their exact resemblance and distinctness. The same holds true of the holding of their identity, which is independent from the holding of their exact resemblance.

As formal features, the identity and distinctness of simple standard tropes are not entities. ${ }^{20}$ They are merely manners of the existence of the $e$ charge, $e$ charge, and $e / 3$ charge. They are neither identical to nor distinct from the $e$ charge, $e$ charge, and $e / 3$ charge. Hence, there just is no need to postulate distinct relata for the identity and distinctness of these tropes, or to construe their identity and distinctness as distinct entities. The relata may be said to be simple and stand in mutually independent and hence arbitrarily different relations.

This result does not compromise the internality of identity or distinctness. If it is assumed that actually $t_{1}=t_{1}=e$ charge, $t_{2}=t_{2}=e$ charge, and $t_{3}=t_{3}=e / 3$ charge, then it can be said that identity is an internal relation in Ehring's terms. Both $t_{1}=t_{1}=e$ charge, $t_{2}=t_{2}=e$ charge, and $t_{3}=t_{3}=e / 3$ charge hold in every

\footnotetext{
${ }^{20}$ Consequently, our response to Ehring differs from Maurin's response to Herbert Hochberg's similar criticism, because she talks about "pseudo-additions" and uses the framework of truth-making (Maurin 2005, 138).
} 
possible world where $t_{1}, t_{2}$, and $t_{3}$ exist, because it is a highly plausible principle that if entities are actually identical, then they are necessarily identical. As was seen just above, $t_{1} \neq t_{2} \neq t_{3}$ holds equally in these worlds on this assumption. Therefore, we may safely conclude that standard tropes can stand in arbitrarily different internal relations and be simple.

Consequently, step 5 in Ehring's argument, which would render $t_{1}, t_{2}$, and $t_{3}$ not simple, is not correct; the hidden premise that entities in distinct arbitrarily different internal relations have parts or multiple, non-identical intrinsic aspects is false. Hence, Ehring's negative conclusion about standard trope nominalism does not follow from his argument. His argument is not sound, as it is premised on an incorrect principle. So, we have argued that the assumption of simple standard tropes does not entail that simple standard tropes are not actually simple. Ehring's argument against the standard view fails. Accordingly, we can restate the standard view by means of the distinction between ontological content and ontological form. First, simple tropes are identified with existing thin natures. According to the standard view, thin natures add something to what exists and to the nature of entities - that is, they contribute to the ontological content of the world. Secondly, being particular, individual, mereologically simple, categorially simple, and being a part, at least, are manners of existence of tropes - their formal features - without being additions of being over and above thin natures. This is constituting the ontological form of the world according to standard trope theory. Formal features of tropes, such as being particular and being individual, are not entities identical to or distinct from thin natures. In contrast to thin natures, tropes are not to be identified with formal features. 


\section{References}

Armstrong, D. M. (1989): Universals - an Opinionated Introduction, Boulder: Westview Press.

Bliss, R.L. (2013): Viciousness and the structure of reality. Philosophical Studies 166 (2): 399-418.

Campbell, K. (1990): Abstract Particulars, Oxford: Blackwell.

Ehring, D. (2011): Tropes, Oxford: Oxford University Press.

Hakkarainen, J. \& Keinänen, M. (2016): “Bradley’s Reductio of Relations and Formal Ontological Relations", in Hemmo Laiho \& Arto Repo (eds.), DE NATURA RERUM - Scripta in honorem professoris Olli Koistinen sexagesimum, Reports from the Department of Philosophy Vol. 38., University of Turku: 246-261.

Hochberg, H. (2004): "Relations, Properties and Predicates", in Relations and Predicates, H. Hochberg and K. Mulligan (eds.), Heusenstamm: Ontos Verlag, 17-53.

Keinänen, M. (2011): “Tropes - the Basic Constituents of Powerful Particulars?”, Dialectica 65 (3).

Keinänen, M. \& Hakkarainen, J. (2010): “Persistence of Simple Substances”, Metaphysica 11 (2): 119135.

Keinänen, M. \& Hakkarainen, J. (2014): “The Problem of Trope Individuation: A Reply to Lowe", Erkenntnis 79 (1): 65-79.

Kriegel, U. (2013): “The Epistemological Challenge of Revisionary Metaphysics”, Philosophers' Imprint 13 (12), < http://hdl.handle.net/2027/spo.3521354.0013.012 >, printed May 6, 2016.

Lowe, E.J. (1998): The Possibility of Metaphysics, Oxford: Oxford University Press.

Lowe, E. J. (2006): The Four-Category Ontology, Oxford: Oxford University Press.

Maurin, A-S. (2002): If Tropes, Dordrecht: Kluwer Academic Publishers.

Maurin, A-S. (2005): “Same but Different”, Metaphysica 6 (1), 131-146. 
Maurin, A-S. (2014), "Tropes", The Stanford Encyclopedia of Philosophy (Fall 2014 Edition), Edward N. Zalta (ed.), URL = http://plato.stanford.edu/archives/fall2014/entries/tropes/, printed May 11, 2016.

Moore, G. E. (1960 [1922]): "External and Internal Relations," in Moore, G.E: Philosophical Studies, London: Routledge and Kegan Paul, 276-309.

Moreland, J. P. (2001): Universals. Montreal: McGill-Queen's University Press.

Mulligan, K. (1998): "Relations - Through Thick and Thin”, Erkenntnis 48, 325-353.

Quine, W. V. (1983): “Ontology and Ideology Revisited”, The Journal of Philosophy 80 (9), 499502.

Quine, W. V. and Ullian, J. S. (1978): The Web of Belief - Second Edition, New York: McGraw-Hill.

Simons, P. (1994): "Particulars in Particular Clothing - Three Trope Theories of Substance", Philosophy and Phenomenological Research LIV: 3, 553-75.

Smith, B. (2005): “Against Fantology", in Experience and Analysis, J. Marek and E. M. Reicher (eds.), Vienna: Austrian Ludwig Wittgenstein Society, 153-170

Smith, B. and Grenon, P. (2004): “The cornucopia of formal-ontological relations”, Dialectica 58 (3), 279-296.

Smith, B. and Mulligan, K. (1983): "Framework for Formal Ontology”, Topoi 2 (1), 73-85.

Smith, B. (1981): “Logic, Form and Matter”, Aristotelian Society Supplementary Volume, 55, 47 74.

Williams, D. C. (1953): “On the Elements of Being I”, Review of Metaphysics 7, 3-18. 